\title{
De novo assembly of Amorpha fruticosa (Amorpha fruticosa $L$.) transcriptome in response to drought stress provides insight into the tolerance mechanisms
}

\author{
Xinzhu SUN \\ Northeast Forestry University \\ He LIU \\ Northest Forestry University \\ Songmiao HU \\ Northeast Forestry University \\ Yunwei ZHOU \\ Jilin Agricultural University \\ Qingjie GUAN ( $\sim$ guanqingjie@nefu.edu.cn ) \\ Northeast Forestry University https://orcid.org/0000-0002-9438-1427
}

\section{Research article}

Keywords: Amorpha fruticosa L., drought stress, transcriptomic analysis, tolerance mechanisms

Posted Date: December 20th, 2019

DOI: https://doi.org/10.21203/rs.2.19443/v1

License: (c) (i) This work is licensed under a Creative Commons Attribution 4.0 International License.

Read Full License 


\section{Abstract}

\section{Background}

Amorpha fruticosa (Amorpha fruticosa L.) is a deciduous shrub that is native to North America and has been introduced to China as an ornamental plant.In order to cultivate drought-tolerant Amorpha fruticosa varieties, it is important to understand the drought-tolerant mechanism of Amorpha fruticosa. Through the changes of the transcriptome of Amorpha fruticosa under drought stress, the mechanism of antistress of Amorpha fruticosa could be revealed. Different concentrations of polyethylene glycol-6000 (PEG-6000) was used to simulate drought stress, and transcriptomic analysis was used to reveal the changes of gene expression patterns in Amorpha fruticosa seedlings.

Results

Results showed that Amorpha fruticosa seedlings were seriously affected by PEG-6000. As for the differently expressed genes (DEGs), most of them were up-regulated. The additional Go and KEGG analysis results showed that DEGs were functionally enriched in cell wall, signal transduction and hormonal regulation related pathways. DEGs like AfSOD, AfHSP, AfTGA, AfbZIP and AfGRX play roles in response to drought stress.

\section{Conclusion}

In conclusion, Amorpha fruticosa seedlings were sensitive to drought, which was different from Amorpha fruticosa tree, and the genes functions in drought stress responses via ABA-independent pathways. The up-regulation of Salicylic acid signal related DEGs (AfTGA and AfPR-1) indicated that Amorpha fruticosa can resist drought stress through Salicylic acid.

\section{Background}

Amorpha fruticosa (Amorpha fruticosa L.) is a deciduous shrub that is native to North America and has been introduced to China as an ornamental plant [1,2]. As a kind of urban greening and slope protection plant, Amorpha fruticosa has high ornamental value and is widely used in the construction of urban landscape and road slope protection. In addition, Amorpha fruticosa also has medicinal value, such as cytotoxic rotenoid glycosides, antibacterial and cytotoxic phenolic metabolite in seeds $[3,4]$.. What more, Amorpha fruticosa leaf was a kind of traditional Chinese medicine used for the treatment of fever, burns, pyogenic carbuncle and eczema $[3,5]$. Amorpha fruticose can tolerate dry soils, but it is most abundant along river banks and roads and the edges of flooded forests, even is tolerant of occasional flooding [6]. The high tolerance of various habitat conditions and potent propagation ability promotes the aggressive invasive behavior of Amorpha fruticosa outside of its native range [6]. In order to breed more drought tolerant Amorpha fruticosa tree varieties, it is important to understand the drought tolerance mechanism in Amorpha fruticosa. Drought stress is one of the most prevalent environmental factors limiting plant growth [7]. Different plants adapt to drought stress in the environment through different mechanisms, but 
most plants could response to drought stress via hormonal regulation, such as abscisic acid (ABA), cytokinin (CK), gibberellic acid (GA), auxin, and ethylene, etc, which regulate diverse processes and enable plant adaptation to drought stress [8,9] Many hormonal regulation related genes had been proved can improve plant resistance, including GH3, NAP, ABIs, AP37, PP2C, PP2C06, PYR/PYL, SIDP366, MYBs, RK1, hox22, SNAC2, OAT, bZIPS, SNAC1, EREBP1, DSM2, AREB2, SRO1c and ABA8OX3 [9]. When plants suffered from stress, a series of biological processes will be induced to respond to stress signals, which will lead to the increase of reactive oxygen species (ROS) content in plant cells [10]. In the long evolutionary process, plants have evolved a series of anti-oxidative system to respond to drought, such as glutathione metabolism pathway, catalase system, peroxidase system, superoxide dismutase system system, etc. As our knowledge, there were several researches found on drought resistance in Amorpha fruticosa. But few articles were focus on the expression pattern and molecular mechanisms of gene action response to drought.

In this study, PEG-6000 was used to simulate drought stress, and transcriptomic analysis was used to reveal the changes of gene expression patterns in Amorpha fruticosa seedlings. The present study will provide theoretical basis and data support for Amorpha fruticosa drought resistant breeding.

\section{Results}

\subsection{Effects of different concentrations of PEG-6000 on plant growth}

After PEG-6000 treatment, the Amorpha fruticosa plants were under drought stress. The leaves of the drought stressed plants showed severe wilting and curling, which were aggravate with the increase of PEG-6000 concentration (Fig. 1). This indicated that Amorpha fruticosa seedlings were sensitive to drought stress, and 30\% concentration PEG-6000 treatment was fatal for plant seedlings. The SOD, MDA, Pro and REC results showed that plants suffered from drought stress had significant higher values than that of CK (Fig. 2).

\subsection{Sequence data summary and de novo assembly}

Approximately $42.6 \mathrm{G}$ raw data including 142 million reads from the six libraries were generated. QC results revealed the good quality of the sequence data with average Q20 of $96.71 \%$, average Q30 of $92.46 \%$, average sequencing error rate of $0.01 \%$ and average GC content of $57.08 \%$ (Table S1). PCA and sample to sample correlation analysis results showed that the biological replicates in each group are clustered together (Fig. $3 a$ and b), which indicated that the variability between samples in a same group

were small. After de novo assembly of clean reads with Trinity, the transcripts were clustering and deredundant, and 96,594 Unigenes were obtained with average length of 864 bp and N50 of 1,430 bp (Table S2). The annotation results showed that 52,010 unigenes were successfully matched with at least one database, including 15,883 (30.54\%), 28,297 (54.41\%), 18,348 (35.28\%), 29,472 (56.67\%), 33,287 
(64.00\%), 28,188 (54.2\%), 46,878 (90.13) and 48,186 (92.65\%) that significantly matched with the COG, GO, KEGG, KOG, Pfam, Swiss-Prot, EggNOG and nr databases, respectively.

\subsection{DEGs between CK and 20\% PEG-6000 treatment group}

There were 1084 and 603 up- and down-regulated DEGs found between CK and 20\% PEG-6000 group. In order to better understand the function of DEGs, we performed GO and KEGG analysis. The GO results showed that regulation of catalytic activity, regulation of peptidase activity, oxidation-reduction process, plant-type cell wall loosening and cell redox homeostasis were the main BP terms respond to drought stress. In which, AfSOD, AfHSP70, AfCYPs, AfCSY4 and 60S ribosomal proteins had high frequency. Most of these DEGs were up-regulated (Fig. 4a). As for KEGG analysis, DEGs were significantly enriched in Starch and sucrose metabolism, Carbon fixation in photosynthetic organisms and 2-0xocarboxylic acid metabolism pathway. In addition, 34 DEGs, including c144508.graph_c0, c164358.graph_c0, c167827.graph_c0, c171717.graph_c1 and c187107.graph_c0, were enriched in plant hormone signal transduction pathway, although the $p$ value $>0.05$. DEGs in plant hormone signal transduction showed that DEGs of salicylic acid downstream were active and transcripts of PP2C were upregulated. Expression of DEGs in ABA and salicylic acid pathway were shown in Fig. $4 \mathrm{~b}$ and c. What more, DEGs in Starch and sucrose metabolism including AfTPS, AfTREH, AfAMY, etc. Most DEGs in Carbon fixation in photosynthetic organisms pathway were up regulated.

\subsection{Interaction network of DEGs and proteins}

Interaction network was performed according to STRING database. There were 262 up-regulated DEGs with Confidence Score $>0.9$ and the interaction network showed that c160801.graph_c0, c192497.graph_c0, c190485.graph_c1, c175551.graph_c1 were the core regulators in these DEGs (Fig. 5a). The expression heatmap showed the distinct gene expression pattern between CK and PEG6000 treatment group (Fig. 5b). The down-regulated DEGs formed an interactive network of six nodes. In addition, we classifid the genes according to their functions, and then construct the interaction network. Genes related to ROS showed a six nodes network (Fig. 5c), of which three genes were differently expressed (Fig. 5d). DEGs related to translation showed that 17 genes had interaction relationship (Fig. 5e) and the expression of which were shown in Fig. $5 \mathrm{f}$.

\section{5 qRT-PCR verification}

A total of 20 genes were verified by qRT-PCR, including AfADH_Zinc_N, AfNAC1, AfZAT10, AfWRKY25, AfPhospholipase A1, AfDREB4, AfRAP2-1, AfABRE3, AfbHLH-MYC, AfABP19a, AfPAP17-X2, AfTrx_2, AfWRKY20, AfMYB1, AfPAL 1, AfMYB2, AfAPX2, AfCAT, AfFe/Mn-SOD and AfGST. The primers used were listed in Table S3. The qRT-PCR results showed that most of the stress respond genes were up-regulated, especially in $20 \%$ and $30 \%$ PEG treatment. Also, some transcription factors were down regulated with the aggravation of stress. All the expression of all these genes was shown in Fig. 6. 


\section{Methods}

\subsection{Plant material and PEG treatment}

Amorpha fruticosa seeds were collected from our research test plot in November 2018 and identified by Seed Key Laboratory of Saline-alkali Vegetation Ecology Restoration, Ministry of Education (Northeast Forestry University). Seeds need to be disinfected before germination test. Amorpha fruticosa seeds were surface-sterilized with $70 \%$ alcohol and $5 \%$ sodium hypochlorite for 5 min followed by rinsing three times with distilled water. Seeds were then seeded into culture bowls full of fine sand (sterilized by high temperature) and cultured in a plant growth chamber (temperature $25^{\circ} \mathrm{C} \pm 2$; relative humidity $60 \% \pm 5$; light intensity $150 \mu \mathrm{mol} \cdot \mathrm{m}^{-2} \mathrm{~s}^{-1}$; light and darkness cycle: 16:8) with sufficient water supply for four weeks. Subsequently, the seedlings were randomly divided into four groups with three repetitions in each group. Osmotic stress was gradually applied with varying concentrations of polyethylene glycol-6000 (PEG6000 ; w/v- $0 \%$, group CK; $10 \%$, group $10 \%$; $20 \%$, group $20 \%$; $30 \%$, group $30 \%$ ) for $72 \mathrm{~h}$. Whole seedlings of all groups were sampled, snap frozen in liquid nitrogen and then stored at $-80{ }^{\circ} \mathrm{C}$ until testing. Superoxide dismutase (SOD), malondialdehyde (MDA), proline (Pro) and relative electrical conductivity (REC) were measured using samples in CK and 20\% group according to previous reports(Guo Q et al.2016; Leonid V et al.2015). Transcriptome sequencing was performed to reveal the gene expression pattern using the six samples in CK and $20 \%$ group. Samples in CK, $10 \%, 20 \%$ and $30 \%$ were used for quantitative real-time PCR (qRT-PCR) detection.

\subsection{RNA extraction, library preparation, and transcriptome sequencing}

Total RNA was isolated using a RNAprep pure Plant Kit (Tiangen, China) according to the manufacturer's instruction. RNA quality was tested using gel electrophoresis, Agilent 2100 (Agilent Technologies Inc., USA) and Nano Drop 2000 (Thermo Fisher Scientifc Inc., USA). Then, total RNA was reverse transcribed to cDNA by a QuantScript RT Kit (Tiangen, China). After that, we started constructing sequencing libraries. An efficient mRNA-seq Library Prep Kit for Illumina (Vazyme, China) was used for the sequence libraries construction. Subsequently, the quality control (QC) was performed by an Agilent 2100 Bioanalyzer and an ABI StepOnePlus Real-Time PCR System to quantify the sample libraries. Finally, all the six mRNA-seq libraries were sequenced on an Illumina HiSeq 4000 sequencing platform with pair-end $2 \times 150$ bp mode to obtain sequencing data. The sequencing data are available at Bigsub database (https://bigd.big.ac.cn/gsub/) with accession number CRA002113.

\subsection{De novo assembly, sequence annotation and differentially expressed genes (DEGs) screening}

Raw reads were filtered to remove adapter and low-quality reads using FasqQC (version 0.11.5) with default parameter settings. De novo transcriptome assembly were performed by Trinity (version 2.2018) using the filtered clean data of the six libraries [13]. The assembled transcripts were hierarchically clustered using Corset (version 1.0.5) [14]. After hierarchical clustering, the longest sequence (unigene) of 
each cluster were used for further analyses, including length distribution statistics, gene annotation ( $\mathrm{Nr}$, $\mathrm{Nt}$, Pfam, KOG/COG, Swiss-prot, KEGG, GO) and identification of DEGs. In addition, ESTScan (version 3.0.2) [15]was used for ORF predication of gene sequences that could not be aligned to any of the abovementioned databases. To evaluate the correlation of biological repetition, principal component analysis (PCA) and pearson's correlation analysis were performed based on the FPKM of reads. Following this, read counts were normalized and DEGs in different comparisons were screened using DEseq2 ( $R$ package) methods [16] with the criteria of padj value $<0.05$ by Negative binomial distribution test and $\mid \log _{2}$ (Fold Change, FC) $\mid \geq 1$.5. Genes with identified as $\log _{2} \mathrm{FC}>1$ and $\log _{2} \mathrm{FC}<-1$ were identified as up- and down-regulated DEGs, respectively. Hierarchical clustering based on the expression profiles of DEGs was presented by pheatmap (version 1.0.10).

\subsection{DEGs functional analysis}

The DEGs enriched into modules correlated with the phenotypes were separately subjected to the enrichment analysis for Gene Ontoloy (GO) and Kyoto Encyclopedia of Genes and Genomes (KEGG) pathways [17]. Significant GO biological processes (BP) and KEGG pathways were identified with the criterion of $p<0.05$. The candidate gene interaction analysis was performed using Cytoscape (version 3.7.2).

\section{5 qRT-PCR verification of RNA-seq data}

qRT-PCR analysis was performed on an Agilent Mx3000P QPCR system (Agilent, USA) using $2 \times$ Brilliant III SYBR Green qPCR Master Mix (Agilent, USA). PCR amplification was performed under the following conditions: $95^{\circ} \mathrm{C}$ for $5 \mathrm{~min}$, followed by 40 cycles of $95^{\circ} \mathrm{C}$ for $30 \mathrm{~s}, 58^{\circ} \mathrm{C}$ for $30 \mathrm{~s}$, and $72^{\circ} \mathrm{C}$ for $30 \mathrm{~s}$ and a final extension at $72^{\circ} \mathrm{C}$ for $5 \mathrm{~min}$. Quantification of gene expression was performed by the comparative 2- $\triangle \Delta C T$ method. The validation analysis was performed with three independent biological replicates. The gene-specific primers for qRT-PCR were designed using Primer Premier 5.0 (http://www.PremierBiosoft.com) and were synthesized by Invitrogen (Carlsbad, USA).

\subsection{Statistical analysis}

Statistical analysis was performed using the GraphPad Prism 8. All experimental data were expressed as mean \pm standard deviation (SD), and differences between groups or treatments were analyzed using the unpaired $t$-test. $P<0.05$ was set as significant threshold for statistical differences.

\section{Discussion}

As an important tree species for highway and slope protection, Amorpha fruticosa is widely used in Northeast China. Amorpha fruticosa is vulnerable to drought stress, so revealing the biological process response to stress is important to Amorpha fruticosa breeding. When environmental conditions become adverse, plants can successfully deploy complex physiological and molecular strategies to cope with abiotic stress [11]. In this study, the curling and wilting of plant leaves prove that plants are under drought stress. The physiological indexes of Amorpha fruticosa against drought stress were consistent with that 
in citrus [18], Tibet Plateau [11] and some shrubs [19]. The tolerance of plants to drought resulted in increasing antioxidants activities which face the increased levels of free radicals [20,21]. The increased contents of SOD, MDA and Pro indicated the cellular defenses against ROS.

The statistical results of DEGs show that most of the DEGs were up-regulated, which demonstrated the response of gene expression pattern to drought stress. The DEGs functional analysis results showed that "regulation of catalytic activity", "regulation of peptidase activity" and "oxidation-reduction process" were the main enriched GO terms and AfSOD, AfHSP70, AfCYPs and 60S ribosomal proteins were the key regulators involved in these terms. SODs are key enzymes in many oxidation processes, and provide basic protection against ROS in plants [22]. The significantly increasing of AfSOD, AfHSP and AfCYPs after PEG-6000 treatment played an important role response to drought stress. Especially, various reports to date reveal that SODs overexpressing plants protect them from oxidative damage [23-26]. Similarly, HSP overexpressing plants also showed excellent stress tolerance [27-29], which act as core genes in our interaction network analysis results. In addition, CYP family members also act as key response genes to drought stress $[30,31]$. The mechanism of improving plant resistance to abiotic stress by SOD, HSP and CYP had been reviewed [32-35], and we will not discuss it in detail. As for KEGG enrichment analysis, we found 34 DEGs enriched in plant hormone signal transduction pathway, including ABA signal related DEGs (AfPP2C, AfPYR/PYL and AfSnRK2) and Salicylic acid signal related DEGs (AfTGA and AfPR-1). ABA is an important signal molecule, which act as a comprehensive factor in response to environmental changes. It play roles the regulation of seed germination and root growth, as well as the adaptive response to various abiotic stresses [36]. In our results, AfPP2C were up-regulated by PEG-6000 treatment. A significant amount of research on PP2C action is related to ABA signaling. The ABA INSENSITIVE1 ( $A B I 1)$ and $A B I 2$ genes encode homologous type-2C protein phosphatases with redundant yet distinct functions in ABA responses [37]. Genetic analysis of abi1 and abi2 mutants, their revertants, transient expression studies, and analysis of transgenic antisense plants showed that PP2Cs act as negative regulators of $A B A$ signaling [38-41]. The increased expression level of AfPP2C revealed that Amorpha fruticosa seedlings were sensitive to drought and the genes functions in drought stress responses via ABA-independent pathways [42]. In addition, AfTGA and AfPR-1 were increased by high concentration of PEG-6000. TGA factors constitute a conserved plant subfamily of basic domain/Leu zipper (bZIP) transcriptional regulators whose genomic targets are thought to include glutathione Stransferase and pathogenesis-related (PR) genes that are associated with detoxification and defense [43-44]. TGA factors contribute to protective gene responses that are mobilized by plants against stress. As for PR-1, of which the expression was promoted by TGA factors [45]. Johnson reported that the in vivo recruitment of TGAs to the PR-1 promoter precedes the SA (Salicylic Acid)-induced expression of a transcriptionally divergent XET gene, which encodes a putative xyloglucan endotransglycosylase [44]. These enzymes remodel the cell wall during development and in response to environmental cues, which is consistent with the enriched cell wall related GO terms in our results. Both AfTGA and AfPR- 1 were upregulated by drought stress, which indicated that Amorpha fruticosa can resist drought stress through SA. The possible mechanism is that when plant suffered from drought stress, NPR1 can transfer the SAinduced activation of PR-1 to the nucleus, where it interacts with the C-terminal domain of TGAs [46]. The 
interaction network analysis results showed that AfHSPs, AfCXXS1, AfGRXS11, AfGRXC6 and AfFAD play roles in gene interaction. Of which, GRX is a kind of small molecule redox protein, which plays an important role in antioxidation in the form of thiol reductase [47]. Pervious study reported that environmental stresses such as cold, heat, drought and salt induce changes in fatty acid composition, mainly in the content of linolenic acid [48]. Zhang found that FAD3 and FAD8 overexpressing tobacco plants showed increased tolerance to drought and to osmotic stress [48]. The FADs induced drought tolerance can be related directly to the changes in membrane structure such as decreased membrane fluidity found in hyperosmotically stressed phospholipid vesicles and yeast cells, and the accumulation of unsaturated fatty acids can attenuate rigidification of membranes leading to reduced damage by osmotic stress [49]. This view was also confirmed in our results, because many genes related to cell wall showed differential expression. The increased AfFAD in Amorpha fruticosa might played similar roles to against to the damage of drought stress.

\section{Conclusions}

Amorpha fruticosa can effectively respond to drought stress by increasing the intracellular content of SOD, MDA and Pro. Correspondingly, the expression of drought stress response genes and transcription factors increased. Some of the transcription factors played key roles in polygenic interaction against to stress. Amorpha fruticosa seedlings were sensitive to drought and the genes functions in drought stress responses via ABA-independent pathways. The up-regulation of Salicylic acid signal related DEGs (AfTGA and AfPR-1) indicated that Amorpha fruticosa can resist drought stress through Salicylic acid.

\section{Additional Files}

Additional files1: Table S1. sequence data summary

Additional files2: Table S2. Unigene assembly imformation

Additional files3: Table S3. Primers imfromation.

\section{Abbreviations}

Go Gene Ontoloy

DEGs Differently expressed genes

KEGG Kyoto Encyclopedia of Genes and Genomes

ABA Abscisic acid

CK cytokinin

GA gibberellic acid 
ROS reactive oxygen species

MDA Malondialdehyde

SOD Superoxide

REC Relative electrical conductivity

PRO Proline

BP Biological processes

PCA principal component analysis

SD standard deviation

\section{Declarations}

\section{Ethics approval and consent to participate}

The authors declare the compliance with institutional, national and international guidelines. The experiment was environmentally friendly and had no negative effects on the local ecosystem.

\section{Consent for publication}

Not Applicable.

\section{Availability of data and materials}

The datasets used and analysed during the current study are available from the corresponding author on reasonable request.

\section{Competing interests}

The authors declare that they have no competing interests. Each of the funding bodies took part in the design of the study and collection, analysis, and interpretation of data, and the writing of the manuscript.

\section{Funding}

The funders had no role in the study design, data collection and analysis, decision to publish, and manuscript preparation.

\section{Authors' contributions}

Q.G. and Y.Z. conceived and designed the study. Q.G., X.S., H.L., and H.S. performed the experiments. Q.G., Y.Z., and X.S. contributed to the sample measurement and data analysis. Q.G.๑S.X. and S.H. wrote the paper. All authors have read and approved the manuscript. 
Acknowledgements

This research was supported by the Natural Science Foundation of Heilongjiang Province of China (C2017009), the National Key Research and Development Program of China (2016YFC0501203), the Fundamental Research Funds for the Central Universities (2572019DF11).

\section{Author details}

1 Key Laboratory of Saline-alkali Vegetation Ecology Restoration Ministry of Education, college of Landscape Architecture, Northeast Forestry University, harbin 150040,china;

2 College of horticulture, Jilin Agricultural University, changchun 130118, China.

\section{References}

1. Hou K: Dictionary of the Famil ies and Genera of Chinese Seed Plants. Beijing: Science Press; 1982.

2. Wang E, Rogel M, Sui X, Chen W, Martínez-Romero E, Berkum P. Mesorhizobium amorphae, a rhizobial species that nodulates Amorpha fruticosa, is native to American soils. Archives of microbiology 2002; 178:301-305.

3. Wu X, Liao H-B, Li G-Q, Liu Y, Cui L, Wu K-F, Zhu X-H, Zeng X-B. Cytotoxic rotenoid glycosides from the seeds of Amorpha fruticosa. Fitoterapia 2014; 100.

4. Muharini R, Díaz A, Ebrahim W, Mándi A, Kurtán T, Rehberg N, Kalscheuer R, Hartmann R, Orfali R, Lin $W$ et al: Antibacterial and cytotoxic phenolic metabolites from the fruits of Amorpha fruticosa, vol. 38; 2017.

5. Hovanet M, Marinas I, Dinu M, Oprea E, Chifiriuc M, Stavropoulou E, Lazar V. The phytotoxicity and antimicrobial activity of Amorpha fruticosa L. leaves extract. Romanian Biotechnological Letters $2015 ; 20: 10670-10678$.

6. Kozuharova E, Matkowski A, Woźniak D, Simeonova R, Naychov Z, Malainer C, Mocan A, Nabavi S, Atanasov A. Amorpha fruticosa - A Noxious Invasive Alien Plant in Europe or a Medicinal Plant against Metabolic Disease? Frontiers in Pharmacology 2017; 8:333.

7. Bray E: Plant Response to Water-deficit Stress. In.; 2007.

8. Wilkinson S, Kudoyarova G, Dmitriy V, Arkhipova T, Davies W. Plant hormone interactions: Innovative targets for crop breeding and management. Journal of experimental botany 2012; 63:3499-3509.

9. Basu S, V R, Kumar A, Pereira A. Plant adaptation to drought stress. F1000Research 2016; 5:1554.

10. Lawlor D, Cornic G. Photosynthetic carbon assimilation and associated metabolism in relation to water deficits in higher plants. Plant, cell \& environment 2002; 25:275-294.

11. Guo Q, Li H, Luo D, Quan H, Bianba D, Zhang WH. Comparative drought tolerance of six native deciduous and broad-leaved woody plant species seedlings in the Qinghai-Tibet Plateau. Acta Physiologiae Plantarum 2016; 38:1-8. 
12. Zhao Y, Aspinall D, Paleg LG. Protection of Membrane Integrity in Medicago sativa L. by Glycinebetaine against the Effects of Freezing. Journal of Plant Physiology 1992; 140:541-543.

13. Chrysant SG, Izzo JL, Jr., Kereiakes DJ, Littlejohn T, 3rd, Oparil S, Melino M, Lee J, Fernandez V, Heyrman R. Efficacy and safety of triple-combination therapy with olmesartan, amlodipine, and hydrochlorothiazide in study participants with hypertension and diabetes: a subpopulation analysis of the TRINITY study. J Am Soc Hypertens 2012; 6:132-141.

14. Davidson NM, Oshlack A. Corset: enabling differential gene expression analysis for de novo assembled transcriptomes. Genome Biol 2014; 15:410.

15. Iseli C, Jongeneel CV, Bucher P. ESTScan: a program for detecting, evaluating, and reconstructing potential coding regions in EST sequences. Proc Int Conf Intell Syst Mol Biol 1999:138-148.

16. Love MI, Huber W, Anders S. Moderated estimation of fold change and dispersion for RNA-seq data with DESeq2. Genome Biol 2014; 15:550.

17. Kanehisa M, Araki M, Goto S, Hattori M, Hirakawa M, Itoh M, Katayama T, Kawashima S, Okuda S, Tokimatsu T. KEGG for linking genomes to life and the environment. Nucleic Acids Research 2007; 36:D480-D484.

18. Hussain S, Khalid M, Saqib M, Ahmad S, Zafar W, Junaid Rao M, Morillon R, Anjum M. Drought tolerance in citrus rootstocks is associated with better antioxidant defense mechanism. Acta Physiologiae Plantarum 2018; 40.

19. Toscano S, Farieri E, Ferrante A, Romano D. Physiological and Biochemical Responses in Two Ornamental Shrubs to Drought Stress. Frontiers in Plant Science 2016; 7.

20. Shao H, Liang ZS, Shao MA. Changes of some anti-oxidative enzymes under soil water deficits among 10 wheat genotypes at maturation stage. Colloids Surf B: Biointerfaces 2005; 45:7-13.

21. Ahmadizadeh M, Nori A, Shahbazi H, Habibpour M. Effects of drought stress on some agronomic and morphological traits of durum wheat (Triticum durum Desf.) landraces under greenhouse condition. African Journal of Biotechnology 2011; 10:14097-14107.

22. Grene R, Erturk N, Heath L. Role of superoxide dismutases (SODs) in controlling oxidative stress in plants. Journal of experimental botany 2002; 53:1331-1341.

23. Badawi G, Yamauchi Y, Shimada E, Sasaki R, Kawano N, Tanaka K, Tanaka K. Enhanced tolerance to salt stress and water deficit by overexpressing superoxide dismutase in tobacco (Nicotiana tabacum) chloroplasts. Plant Science 2004; 166:919-928.

24. Rubio M, González E, Minchin F, Webb J, Arrese-Igor C, Ramos J, Becana M. Effects of water stress on antioxidant enzymes of leaves and nodules of transgenic alfalfa overexpressing superoxide dismutases. Physiologia plantarum 2002; 115:531-540.

25. Zlatev Z, Lidon F, Ramalho J, Yordanov I. Comparison of resistance to drought of three bean cultivars. Biologia Plantarum 2006; 50:389-394.

26. Wang C, Yang A, Yin H, Zhang J. Influence of Water Stress on Endogenous Hormone Contents and Cell Damage of Maize Seedlings. Journal of integrative plant biology 2008; 50:427-434. 
27. Wang X, Yan B, Shi M, Zhou W, Zekria D, Wang H, Kai G. Overexpression of a Brassica campestris HSP70 in tobacco confers enhanced tolerance to heat stress. Protoplasma 2015; 253.

28. Xiang J, Chen X, Hu W, Xiang Y, Yan M, Wang J. Overexpressing heat-shock protein OsHSP50.2 improves drought tolerance in rice. Plant Cell Reports 2018; 37.

29. Wang A, Yu X, Mao Y, Liu Y, Liu G, Liu Y, Niu X. Overexpression of a small heat-shock-protein gene enhances tolerance to abiotic stresses in rice. Plant Breeding 2015; 134:n/a-n/a.

30. Sekhar K, Bhyri P, Vudem D, Khareedu V. Isolation and characterization of a pigeonpea cyclophilin (CcCYP) gene, and its over-expression in Arabidopsis confers multiple abiotic stress tolerance. Plant, cell \& environment 2010; 33:1324-1338.

31. Duan F, Ding J, Lee D, Lu X, Feng Y-Q, Song W. Overexpression of SoCYP85A1, a Spinach Cytochrome p450 Gene in Transgenic Tobacco Enhances Root Development and Drought Stress Tolerance. Frontiers in Plant Science 2017; 8:1909.

32. Kim Y-H, Khan A, Waqas M, Lee I-J. Silicon Regulates Antioxidant Activities of Crop Plants under Abiotic-Induced Oxidative Stress: A Review. Frontiers in Plant Science 2017; 8.

33. Verma G, Srivastava D, Tiwari P, Chakrabarty D: ROS Modulation in Crop Plants Under Drought Stress. In.; 2019: 311-336.

34. Park C-J, Seo Y-S. Heat Shock Proteins: A Review of the Molecular Chaperones for Plant Immunity. The plant pathology journal 2015; 31:323-333.

35. Whitley D, Goldberg S, Jordan W. Heat shock proteins: A review of the molecular chaperones. Journal of vascular surgery : official publication, the Society for Vascular Surgery [and] International Society for Cardiovascular Surgery, North American Chapter 1999; 29:748-751.

36. Fujii H, Chinnusamy V, Rodrigues A, Rubio S, Antoni R, Park S-Y, Cutler SR, Sheen J, Rodriguez PL, Zhu J-K. In vitro reconstitution of an abscisic acid signalling pathway. Nature 2009; 462:660-664.

37. Chak R, Thomas T, Quatrano R, Rock $C$. The genes $A B I 1$ and $A B I 2$ are involved in abscisic acid- and drought-inducible expression of the Daucus carota L. Dc3 promoter in guard cells of transgenic Arabidopsis thaliana (L.) Heynh. Planta 2000; 210:875-883.

38. Rodriguez P. Protein phosphatase 2C (PP2C) function in high plants. Plant molecular biology 1999; 38:919-927.

39. Sheen J. Mutational Analysis of Protein Phosphatase 2C Involved in Abscisic Acid Signal Transduction in Higher Plants. Proceedings of the National Academy of Sciences of the United States of America 1998; 95:975-980.

40. Merlot S, Gosti F, Guerrier D, Vavasseur A, Giraudat J. The ABI1 and ABI2 protein phosphatases 2C act in a negative feedback regulatory loop of the abscisic acid signalling pathway. The Plant journal : for cell and molecular biology 2001; 25:295-303.

41. Gosti F, Beaudoin N, Serizet C, Webb A, Vartanian N, Giraudat J. ABI1 Protein Phosphatase 2C Is a Negative Regulator of Abscisic Acid Signaling. The Plant cell 1999; 11:1897-1910. 
42. Yoshida T, Mogami J, Yamaguchi-Shinozaki K. ABA-dependent and ABA-independent signaling in response to osmotic stress in plants. Current opinion in plant biology 2014; 21C:133-139.

43. Klinedinst S, Pascuzzi P, Redman J, Desai M, Arias J. A xenobiotic-stress-activated transcription factor and its cognate target genes are preferentially expressed in root tip meristems. Plant Molecular Biology 2000; 42:679-688.

44. Johnson C, Boden E, Arias J. Salicylic Acid and NPR1 Induce the Recruitment of trans-Activating TGA Factors to a Defense Gene Promoter in Arabidopsis. The Plant cell 2003; 15:1846-1858.

45. Gatz C. From Pioneers to Team Players: TGA Transcription Factors Provide a Molecular Link Between Different Stress Pathways. Molecular plant-microbe interactions : MPMI 2012; 26.

46. Kinkema M, Fan W, Dong X. Nuclear Localization of NPR1 Is Required for Activation of PR Gene Expression. The Plant cell 2001; 12:2339-2350.

47. Noctor G, Reichheld J-P, Foyer C. ROS-related redox regulation and signaling in plants. Seminars in Cell \& Developmental Biology 2017; 80.

48. Zhang M, Barg R, Yin M, Gueta-Dahan Y, Leikin-Frenkel A, Salts Y, Shabtai S, Ben-Hayyim G. Modulated fatty acid desaturation via overexpression of two distinct $\omega-3$ desaturases differentially alters tolerance to various abiotic stresses in transgenic tobacco cells and plants. The Plant journal : for cell and molecular biology 2005; 44:361-371.

49. Mikami K, Murata N. Membrane fluidity and the perception of environmental signals in cyanobacteria and plants. Progress in lipid research 2003; 42:527-543.

\section{Figures}

\section{Figure 1}

Growth of Amorpha fruticosa seedlings under different drought stress conditions. $0 \%$ means control group without PEG-6000 treatment; $10 \%$ means PEG-6000 treatment group, $\mathrm{w} / \mathrm{v}=10 \% ; 10 \%, 20 \%$ and $30 \%$ means PEG-6000 treatment group, $w / v=10 \%, w / v=20 \%$ and $w / v=30 \%$, respectively.

\section{Figure 2}

Contents of SOD, MDA, Pro and REC under drought stress. Abscissa shows the index and unit. Ordinate numbers represent values

\section{Figure 3}


Clustering results of all the samples. a. Principal component analysis (PCA) clustering results. b. The sample-to-sample clustering analysis based on the mRNA expression level. The color depth notes the similarity between samples (0 1). The deeper the color, the higher the similarity.

\section{Figure 4}

DEGs expression pattern and candidate pathways. a. DEGs expression patterns in different samples. $b$. DGEs expression pattern in ABA signaling pathway. c. DEGs expression pattern in Salicylic acid signaling pathway. 

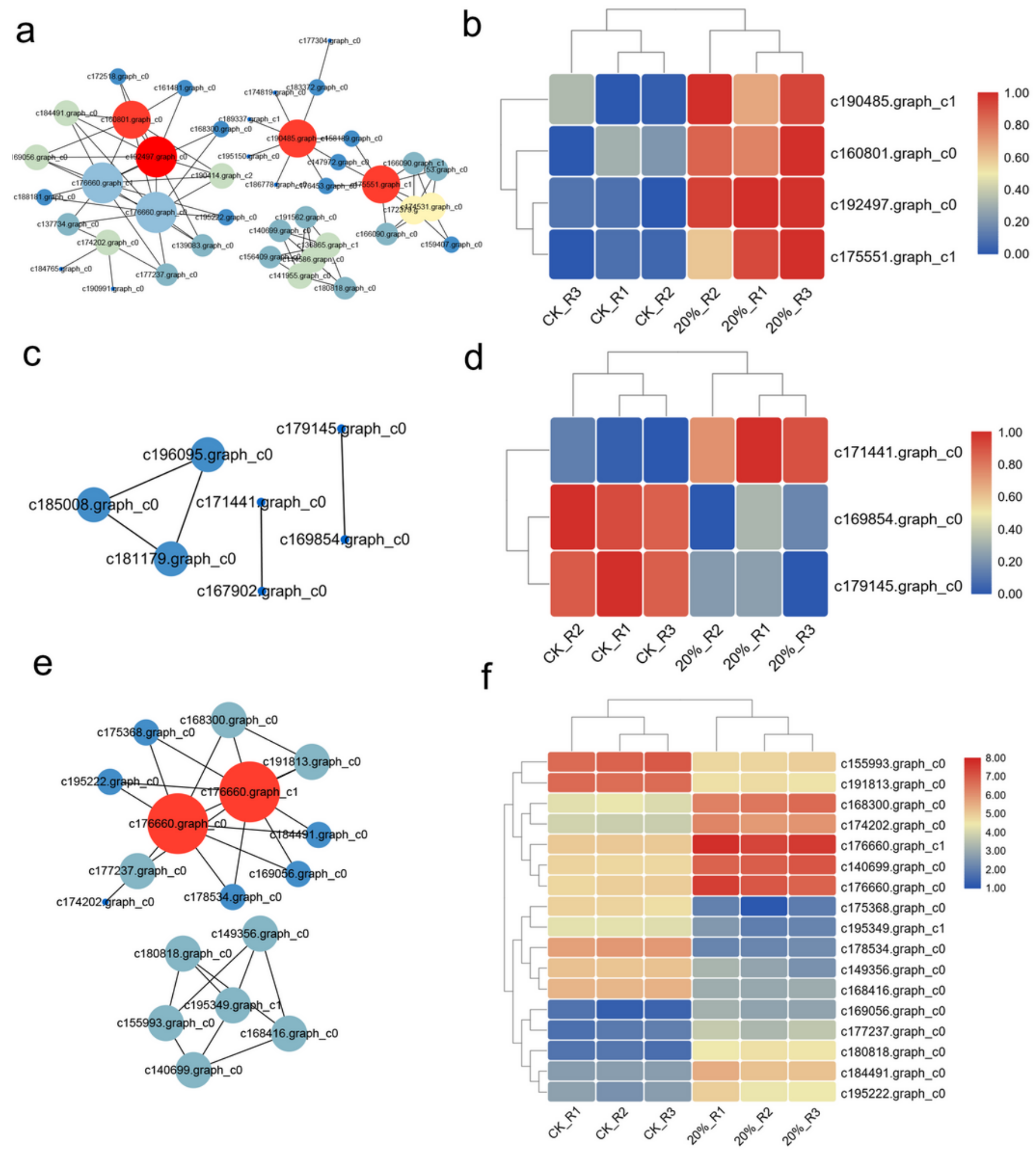

\section{Figure 5}

Interaction network of DEGs and proteins. a. interaction network of up-regulated DEGs under drought stress. b. Heatmap of core regulators in the interaction network of a. c. Interaction network of genes related to ROS. d. Heatmap of core regulators in the interaction network of b. e. Interaction network of genes related to translation. f. Heatmap of core regulators in the interaction network of e. 
Figure 6

qRT-PCR verification results.

\section{Supplementary Files}

This is a list of supplementary files associated with this preprint. Click to download.

- TableS1sequencedatasummary.xlsx

- TableS3primersimfromation.xlsx

- TableS2Unigeneassemblyimformation.xlsx 